Editorial

\title{
Biomarkers of Pelvic Endometriosis
}

\section{Biomarcadores de endometriose pélvica}

\author{
Fernando Marcos dos Reis ${ }^{1}$ Cecília de Souza Monteiro ${ }^{1}$ Márcia Mendonça Carneiro ${ }^{1}$ \\ 1 Division of Human Reproduction, Hospital das Clínicas, Department \\ of Obstetrics and Gynecology, Universidade Federal de Minas Gerais, \\ Belo Horizonte, MG, Brazil
}

Rev Bras Ginecol Obstet 2017;39:91-93.

Endometriosis is typically a symptomatic disease, and the symptoms often manifest as dysmenorrhea, dyspareunia, chronic pelvic pain, and/or infertility. Deep infiltrating endometriosis can also produce cyclic urinary or intestinal complaints. Nevertheless, the symptoms of endometriosis are not specific, and may be associated with many other different conditions. Severe dysmenorrhea in adolescent girls may be underestimated by health care providers and accepted as a physiological fate. Thus, patients can spend years without the accurate diagnosis and the proper treatment, with a tremendous negative impact on their quality of life. ${ }^{1}$

The use of a non-invasive biomarker would bring a remarkable advance to the management of endometriosis. A good test could shorten the time lapse between the onset of the symptoms and the beginning of the treatment, and allow monitoring of disease progression and recurrence. ${ }^{2}$ Imaging methods such as transvaginal ultrasound and magnetic resonance allow the precise anatomical localization of some forms of endometriosis., ${ }^{3,4}$ Although accurate for ovarian endometrioma and deep infiltrating lesions, these imaging methods are operator-dependent, require a highly specialized training, and fail to detect superficial peritoneal endometriotic implants. ${ }^{5,6}$

Decades of research have not led to a reliable biomarker for the non-invasive detection of endometriosis. The intriguing question is not "why have we failed," but "why should we have succeeded" in this endeavor. Do we have a good serum marker for breast cancer, coronary heart disease, or osteoporosis? Like these and many other prevalent and disabling conditions, endometriosis is a focal disease with some systemic features and biochemical signs that are too nonspecific to be accurately used as diagnostic biomarkers.

No endometriosis-specific antigen has been discovered so far, nor endometrium-specific molecules to be traced in search for ectopic endometrial implants. Endometriotic lesions are often small, have scarce cellular content, and usually do not release inflammatory mediators or growth factors in amounts that outweigh alternative sources of the same molecules. At best, some patients have increased serum levels of putative endometriosis products, but there is always a contingent of women with confirmed disease and normal serum marker levels, which confers low sensitivity (high false negative rate) to the method.

Currently, no serum biomarker is validated as a diagnostic test for endometriosis. A recent Cochrane review retrieved 141 studies that evaluated 122 serum or plasma biomarkers of endometriosis in more than 15,000 subjects. Meta-analysis was only possible for cancer antigen (CA)-125, CA-19.9, anti-endometrial antibodies and interleukin-6. The disappointing conclusion is that none of the evaluated biomarkers was accurate enough to be used in the daily practice. ${ }^{7}$ The most studied of these proteins is CA-125, a glycoprotein produced by endometrial and mesothelial cells in response to inflammation. ${ }^{8}$ The concentrations of CA-125 vary across the menstrual cycle, being higher during menstruation and lower in the follicular and ovulatory phases. The magnitude of the CA-125 increase during menstruation is amplified in women with endometriosis. However, CA-125 is not specific for endometriosis, and has low sensitivity for disease detection at any stage. ${ }^{2}$ Therefore, it is not currently recommended as a diagnostic tool for endometriosis.

There are, however, some encouraging perspectives. High-throughput molecular studies have opened an avenue to the rational discovery of molecules that are overexpressed in endometriotic lesions and/or in the eutopic endometrium of patients with the disease. ${ }^{9-11}$ This approach led to the discovery of five micro-RNAs with increased expression in peritoneal endometriotic lesions compared with healthy surrounding tissues. ${ }^{9}$ Another study found 214 proteins differentially expressed in ovarian endometrioma versus eutopic endometrium from the same patients. ${ }^{10}$ Performing
Address for correspondence Fernando M. Reis, MD, PhD, Universidade Federal de Minas Gerais, Belo Horizonte, MG, Brazil 14048-900

(e-mail: reis.ufmg@gmail.com).
DOI http://dx.doi.org/ 10.1055/s-0037-1601398. ISSN $0100-7203$.
Copyright @ 2017 by Thieme-Revinter Publicações Ltda, Rio de Janeiro, Brazil
License terms

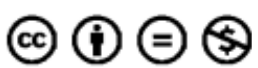



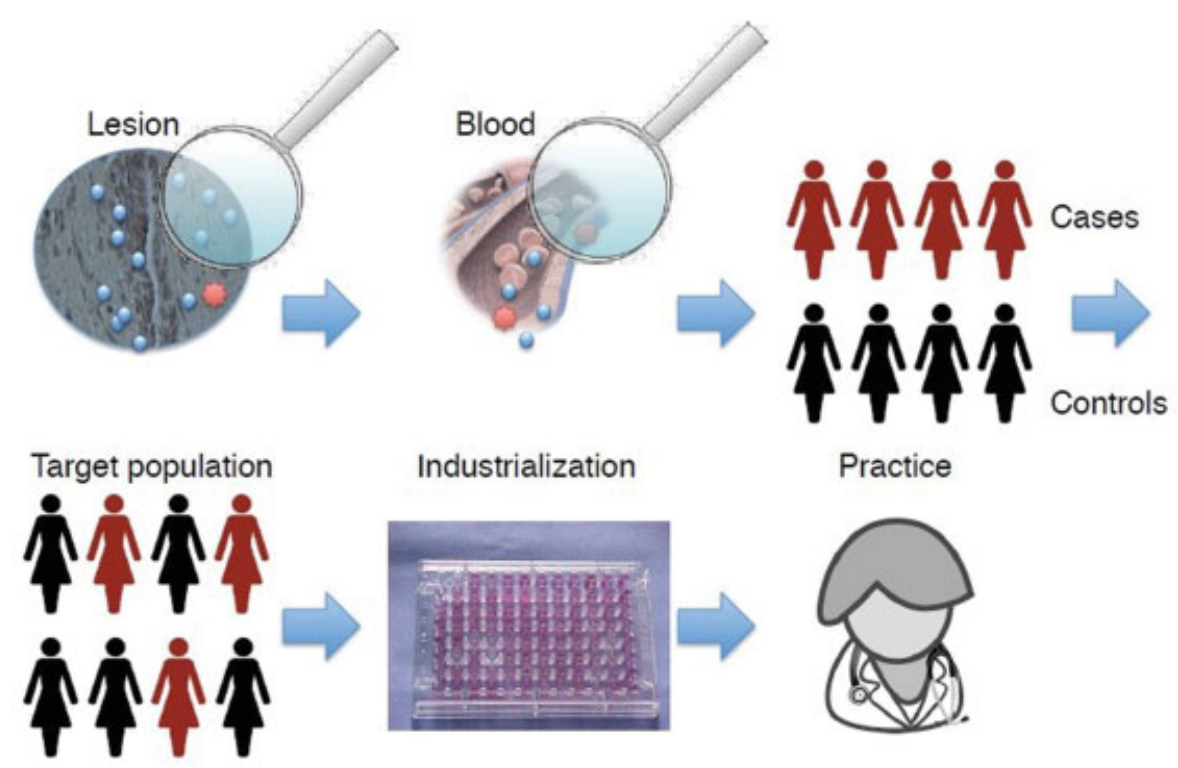

Fig. 1 Steps of endometriosis biomarker discover and validation before industrial production and routine use.

proteomic studies directly in the peripheral blood is an obvious shortcut to the discovery of new serum analytes that may be consistently altered in women with endometriosis. ${ }^{12}$ However, this strategy is more vulnerable to noise from abundant serum proteins that mask the proteins of interest, ${ }^{13}$ and has not yet revealed protein markers with diagnostic utility in endometriosis.

Crossing the bridge from bench to bedside remains a challenge for researchers in the field (-Fig. 1). First, experimental studies to select candidate markers based on unique pathophysiological mechanisms or large proteomic or metabolomic profiles should be performed. Second, test validation in the preclinical context, including the comparison between volunteers with an established diagnosis and a healthy control group should be made. Third, studies in the target population to assess the test performance and calculate its predictive value among individuals with unknown diagnoses, such as women with symptoms suggestive of endometriosis, must be performed. Last but not least, the industrial development of the test for clinical use must be set up. ${ }^{14}$ Once these steps are completed, it will be time to define when and to whom the test will be applied. ${ }^{15,16}$ Does it make sense to perform an endometriosis blood test in all infertile women, or in all cases of pelvic pain? Should the test be used for diagnosis, screening, or both?

To conclude, the saga of endometriosis biomarkers enters the year of 2017 still confined to the research territory, and it may take some additional time to cross the border of evidence-based clinical practice. Meanwhile, the constant improvement of imaging techniques and the recognition that endometriosis can be medically treated based on strong clinical suspicion ${ }^{16}$ may allow earlier medical care and, if necessary, well planned, timely and thorough surgical intervention to relieve symptoms and improve the patients' quality of life.

\section{Acknowledgments}

Research in the authors' laboratory is supported by Conselho Nacional de Desenvolvimento Científico e Tecnológico (CNPq, grant \# 311154/2015-8).

\section{References}

1 Yeung P, Gupta S, Gieg S. Endometriosis in adolescents: a systematic review. J Endometr Pelvic Pain Disord. 2017. Doi: $10.5301 /$ je. 5000264

2 May KE, Conduit-Hulbert SA, Villar J, Kirtley S, Kennedy SH, Becker CM. Peripheral biomarkers of endometriosis: a systematic review. Hum Reprod Update 2010;16(06):651-674

3 Abrao MS, Gonçalves MO, Dias JA Jr, Podgaec S, Chamie LP, Blasbalg R. Comparison between clinical examination, transvaginal sonography and magnetic resonance imaging for the diagnosis of deep endometriosis. Hum Reprod 2007;22(12):3092-3097

4 Manganaro L, Anastasi E, Vinci V, et al. Endometriosis: 10 keys points for MRI. J Endometr Pelvic Pain Disord. 2015;7(01):10-18

5 Tammaa A, Fritzer N, Strunk G, Krell A, Salzer H, Hudelist G. Learning curve for the detection of pouch of Douglas obliteration and deep infiltrating endometriosis of the rectum. Hum Reprod 2014;29(06):1199-1204

6 Holland TK, Cutner A, Saridogan E, Mavrelos D, Pateman K, Jurkovic D. Ultrasound mapping of pelvic endometriosis: does the location and number of lesions affect the diagnostic accuracy? A multicentre diagnostic accuracy study. BMC Womens Health 2013;13:43

7 Nisenblat V, Bossuyt PM, Shaikh R, et al. Blood biomarkers for the non-invasive diagnosis of endometriosis. Cochrane Database Syst Rev 2016;(05):CD012179

8 Fassbender A, Vodolazkaia A, Saunders P, et al. Biomarkers of endometriosis. Fertil Steril 2013;99(04):1135-1145

9 Saare M, Rekker K, Laisk-Podar T, et al. High-throughput sequencing approach uncovers the miRNome of peritoneal endometriotic lesions and adjacent healthy tissues. PLoS One 2014;9(11): e112630

10 Vehmas AP, Muth-Pawlak D, Huhtinen K, et al. Ovarian endometriosis signatures established through discovery and directed mass spectrometry analysis. J Proteome Res 2014;13(11):4983-4994 
11 Laudański P, Szamatowicz J, Oniszczuk M. Profiling of peritoneal fluid of women with endometriosis by chemokine protein array. Adv Med Sci 2006;51:148-152

12 Seeber B, Sammel MD, Fan X, et al. Proteomic analysis of serum yields six candidate proteins that are differentially regulated in a subset of women with endometriosis. Fertil Steril 2010;93(07): 2137-2144

13 Meehan KL, Rainczuk A, Salamonsen LA, Stephens AN. Proteomics and the search for biomarkers of female reproductive diseases. Reproduction 2010;140(04):505-519
14 Fassbender A, Burney ROO DF,D'Hooghe T, Giudice L. Update on Biomarkers for the Detection of Endometriosis. BioMed Res Int 2015;2015:130854

15 Somigliana E, Vercellini P, Vigano' P, Benaglia L, Crosignani PG, Fedele L. Non-invasive diagnosis of endometriosis: the goal or own goal? Hum Reprod 2010;25(08):1863-1868

16 Vercellini P, Giudice LC, Evers JL, Abrao MS. Reducing lowvalue care in endometriosis between limited evidence and unresolved issues: a proposal. Hum Reprod 2015;30(09): 1996-2004 